\title{
-NOTES-
}

\section{NONLINEAR SURFACE WAVES IN SELF-GRAVITATING FLUIDS*}

\author{
By S. K. MALIK AND M. SINGH (Simon Fraser University)
}

\begin{abstract}
The weakly nonlinear disturbances in a self-gravitating, incompressible, inviscid fluid slab are studied. When the wave number is equal to the critical wave number, the amplitude modulation results in nonlinear Schrödinger equation. The finiteamplitude standing wave is stable against modulation. The nonlinear cutoff wave number is also obtained.
\end{abstract}

1. Introduction. The linear analysis employed by Oganesian [1] and Chakraborty [2] to investigate the stability of a self-gravitating slab shows that the system is gravitationally unstable for wave numbers (normalized with respect to the width of the slab) less than the critical wave number $k_{c}=0.6392$.

Tassoul and Dedic [3] examined the nonlinear stability for such a problem. However, they did not take into account amplitude modulation. In this presentation, we consider the nonlinear stability of a self-gravitating slab against modulation using the multiple time scale method. We have shown that the waves are modulationally stable against the standing wave. The nonlinear Schrödinger equation governs the amplitude modulation such that the solitary waves, phase jumps, and wave train of constant amplitude are just the special cases.

2. Formulation. An invisid incompressible self-gravitating fluid slab of finite thickness is considered. There is a vacuum on both sides of the slab. The slab is assumed to extend infinitely in both the $x$ and $y$ directions and to be confined between the planes $|z|=Z$. We assume that the flow field due to wave motion is irrotational. The basic equations governing self-gravitating hydrodynamics in Cartesian coordinates are:

$$
\begin{gathered}
\nabla^{2} \phi=\left(\frac{\partial^{2}}{\partial x^{2}}+\frac{\partial^{2}}{\partial z^{2}}\right) \phi(x, z)=0, \\
\nabla^{2} v(x, z)=1,
\end{gathered}
$$

and

$$
\nabla^{2} w(x, z)=0,
$$

for $0 \leq|z| \leq 1+\eta(x, t)$ and $-\infty<x<\infty$ where $t$ denotes time and $\eta$ the elevation of the free surface measured from the unperturbed level. We shall discuss only the twodimensional problem.

* Received July 23, 1979; revised version received November 27, 1979. 
Here $\phi(x, z), v(x, z)$, and $w(x, z)$ represent the velocity, internal and external gravitational potentials, respectively. All quantities are normalized with respect to the characteristic length $Z$ and the characteristic time $\left(4 \pi G \rho_{0}\right)^{-1 / 2}$, where $G$ is the constant of gravitation and $\rho_{0}$ the density. The wave number $k$ is measured in units of $1 / Z$. The slab is in hydrostatic equilibrium in which the equilibrium surfaces are $|z|=1$. At the free surfaces $|z|=1+\eta(x, t)$, the boundary conditions are:

$$
\begin{gathered}
\frac{\partial \eta}{\partial t}+\frac{\partial \eta}{\partial x} \frac{\partial \phi}{\partial x}-\frac{\partial \phi}{\partial z}=0 \\
\frac{\partial \phi}{\partial t}+\frac{1}{2}\left[\left(\frac{\partial \phi}{\partial x}\right)^{2}+\left(\frac{\partial \phi}{\partial z}\right)^{2}\right]+v=0 \\
\partial v / \partial z=\partial w / \partial z \\
\partial v / \partial x=\partial w / \partial x
\end{gathered}
$$

We study the self-modulation of weakly nonlinear quasi-monochromatic waves with narrow band spectrum. To that purpose we employ the method of multiple scales by expanding $\phi, \eta, v$ and $w$ as asymptotic series in terms of a small parameter $\varepsilon$. This leads to linear and successive nonlinear partial differential equations of various orders.

3. Linear theory. Since there is no steady flow in the unperturbed state, the zerothorder problem yields the following solutions:

$$
\phi_{0}=0, \quad v_{0}=\frac{1}{2} z^{2}, \quad w_{0}=z .
$$

For the first-order problem, we choose the following quasi-monochromatic wave as the initial solution:

$$
\begin{gathered}
\eta_{1}=A\left(x_{1}, x_{2} ; t_{1}, t_{2}\right) \exp (i \psi)+\text { c.c, } \\
\phi_{1}=\frac{-i \omega \cosh k z}{k} \frac{\sinh k}{\sinh }\left\{A\left(x_{1}, x_{2} ; t_{1}, t_{2}\right) \exp (i \psi)\right. \\
\left.-\bar{A}\left(x_{1}, x_{2} ; t_{1}, t_{2}\right) \exp (-i \psi)\right\}+B_{1}\left(x_{1}, x_{2} ; t_{1}, t_{2}\right), \\
v_{1}=-\frac{\cosh k z}{k(1+\operatorname{tgh} k) \cosh k} A \exp (i \psi)+\text { c.c }+B_{2}\left(x_{1}, x_{2} ; t_{1}, t_{2}\right), \\
w_{1}=-\frac{1}{k(1+\operatorname{tgh} k)} A \exp (i \psi) \exp (-k(|z|-1))+\text { c.c } \\
+B_{3}\left(x_{1}, x_{2} ; t_{1}, t_{2}\right),
\end{gathered}
$$

where

$$
\psi=k x_{0}-\omega t_{0} .
$$

Here c.c stands for complex conjugate. The constants $B_{1}, B_{2}$ and $B_{3}$ are assumed to be real and independent of the lowest scales $x_{0}, t_{0}$, and reveal the arbitrariness associated with the velocity and gravitational potentials, respectively. In order that the starting 
solutions be nontrivial, the frequency $\omega$ and wave number $k$ must satisfy the dispersion relation:

$$
\left.\omega^{2}=k \operatorname{tgh} k \mid 1-\frac{1}{k(1+\operatorname{tgh} k)}\right\} \text {, }
$$

with

$$
B_{2}=0 \text {. }
$$

The traveling wave train solutions are possible only for $k \geq k_{c}$, where $k_{c}$ is the solution of the transcendental equation

$$
k(\operatorname{tgh} k+1)=1,
$$

which yields

$$
k_{c}=0.6392 .
$$

For $k<k_{c}$, the fluid flow is obviously unstable.

4. Amplitude modulation of the standing wave. We first proceed to the second-order problem. As we are interested in waves near $k=k_{c}$ and $\omega=0$, the carrier wave is a standing wave. The non-secularity condition for $\eta_{2}$ is

$$
\partial A / \partial x_{1}=0,
$$

implying that the amplitude is independent of the fast variable $x_{1}$. The solutions of the second-order problem thus become

$$
\begin{gathered}
\phi_{2}=B_{4}\left(x_{1}, x_{2} ; t_{1}, t_{2}\right), \\
\eta_{2}=-\frac{\partial B_{1}}{\partial t_{1}}-B_{5}+|A|^{2}\left(1-2 k_{c}\right)+q_{1} A^{2} \exp \left(2 i \psi_{1}\right)+\text { c.c }, \\
v_{2}=q_{2} A^{2} \frac{\cosh 2 k_{c} z}{\cosh 2 k_{c}} \exp \left(2 i \psi_{1}\right)+\text { c.c }+B_{5}\left(x_{1}, x_{2} ; t_{1}, t_{2}\right), \\
w_{2}=q_{3} \exp \left(-2 k_{c}(|z|-1)\right) \exp \left(2 i \psi_{1}\right)+\text { c.c }+B_{6}\left(x_{1}, x_{2} ; t_{1}, t_{2}\right),
\end{gathered}
$$

where

$$
\begin{gathered}
\psi_{1}=k_{c} x_{0}, \quad q_{1}=k_{c}-\frac{1}{2} q_{4} \frac{\left(4 k_{c}-1\right)}{q_{4}-1}, \\
q_{2}=\frac{1}{q_{4}}\left(k_{c}-q_{1}\right), \quad q_{3}=q_{2}-\frac{A^{2}}{2}, \\
q_{4}=2 k_{c}\left(\operatorname{tgh} 2 k_{c}+1\right) .
\end{gathered}
$$

Here $B_{4}, B_{5}$ and $B_{6}$ are constants of the lower scales.

In order to obtain the amplitude of standing wave, we now consider the third-order problem:

$$
L_{0}\left(\phi_{3}\right)=-\left(\partial^{2} B_{1} / \partial x_{1}^{2}\right),
$$




$$
\begin{aligned}
& L_{0}\left(v_{3}\right)=-2 \frac{\partial^{2} v_{2}}{\partial x_{0} \partial x_{1}}-\frac{\partial^{2} v_{1}}{\partial x_{1}^{2}}-2 \frac{\partial^{2} v_{1}}{\partial x_{0} \partial x_{2}} \\
& L_{0}\left(w_{3}\right)=-2 \frac{\partial^{2} w_{2}}{\partial x_{0} \partial x_{1}}-\frac{\partial^{2} w_{1}}{\partial x_{1}^{2}}-2 \frac{\partial^{2} w_{1}}{\partial x_{0} \partial x_{2}}
\end{aligned}
$$

where the linear operator $L$ is defined to be

$$
L_{0}=\left(\frac{\partial^{2}}{\partial x_{0}^{2}}+\frac{\partial^{2}}{\partial z^{2}}\right) .
$$

The boundary conditions (4)-(7) at $|z|=1$ now take the form:

$$
\begin{aligned}
& \frac{\partial \eta_{3}}{\partial t_{0}}-\frac{\partial \phi_{3}}{\partial z}= \frac{\partial}{\partial t_{1}}\left(\frac{\partial B_{1}}{\partial t_{1}}-\left(1-2 k_{c}\right)|A|^{2}\right) \\
&+ \text { terms involving } \exp \left( \pm i \psi_{1}\right) \text { and } \exp \left( \pm 2 i \psi_{1}\right) \\
& \frac{\partial \phi_{3}}{\partial t_{0}}+v_{3}+\eta_{3}= A^{2} \bar{A}\left(-2 k_{c} q_{2} \operatorname{tgh} 2 k_{c}-k_{c} q_{1}+\frac{3}{2} k_{c}^{2}\right) \exp \left(i \psi_{1}\right) \\
&-\frac{1}{\left(1-k_{c}\right)} \frac{\partial^{2}}{\partial t_{1}^{2}} A \exp \left(i \psi_{1}\right)-k_{c} A \\
& \times\left(-\frac{\partial B_{1}}{\partial t_{1}}+|A|^{2}\left(1-2 k_{c}\right)\right) \exp \left(i \psi_{1}\right) \\
&+ \mathrm{c.c}+\operatorname{terms} \text { involving } \exp \left( \pm 2 i \psi_{1}\right) \text { and } \exp \left( \pm 3 i \psi_{1}\right), \\
& \frac{\partial v_{3}}{\partial x_{0}}-\frac{\partial w_{3}}{\partial x_{0}}=A^{2} \bar{A} q_{1} \exp \left(i \psi_{1}\right)+\mathrm{c.c}+\text { terms involving } \\
& \frac{\partial v_{3}}{\partial z}-\frac{\partial w_{3}}{\partial z}+ \eta_{3}=A^{2} \bar{A} k^{2} \exp \left(i \psi_{1}\right)+\mathrm{c.c}+\text { terms involving } \\
& \exp \left( \pm 2 i \psi_{1}\right) \text { and } \exp \left( \pm 3 i \psi_{1}\right) .
\end{aligned}
$$

We assume that $B_{1}, B_{2}$ and $B_{3}$ depend upon the lower scale only through $A$ so that they are independent of $x_{1}$ by virtue of (29). Since the left-hand side of (34) produces no "constant" term, we have

$$
\frac{\partial B_{1}}{\partial t_{1}}=|A|^{2}\left(1-2 k_{c}\right)+E\left(x_{2}, t_{2}\right),
$$

where $E\left(x_{2}, t_{2}\right)$ is a constant to be determined by the initial conditions with respect to $t_{1}$. To eliminate secular behavior, we need to consider only terms involving $\exp \left(i \psi_{1}\right)$ in Eqs. (35)-(37):

$$
i \frac{\partial A}{\partial x_{2}}+P \frac{\partial^{2} A}{\partial t_{1}^{2}}=Q|A|^{2} A+R A
$$


where

$$
\begin{gathered}
P=-\frac{1}{2}\left(\frac{\partial^{2} k}{\partial \omega^{2}}\right)_{k=k_{c}}=-\frac{1}{2\left(1-k_{c}\right)}, \\
Q=-\frac{k_{c}^{3}\left(4 k_{c}-1\right)}{4 k_{c}-2 k_{c}^{2}-1}, \quad R=-\frac{1}{2} k_{c} E .
\end{gathered}
$$

Eq. (39) is a nonlinear Schrödinger equation with time and space variables interchanged and hence furnishes the amplitude modulation of standing waves. It turns out that the linear interaction term causes only phase shift and thus can be removed by using the transformation

$$
A\left(x_{2}, t_{1}\right)=A \exp \left(-i \int^{x_{2}} R\left(x_{2}^{\prime}\right) d x_{2}^{\prime}\right)
$$

With $P=-0.7216$ and $Q=-0.860, P Q>0$ and therefore finite-amplitude plane wave is stable against modulation (Whitham [4]).

We now examine the plane wave solution of the form

$$
A\left(x_{2}, t_{1}\right)=A_{0} \exp \left(i\left(k x_{2}-\Omega t_{1}\right)\right),
$$

where $A_{0}$ is constant. On substituting (44) into (39), we get the dispersion relation

$$
\Omega^{2}=-\left(\frac{K+Q\left|A_{0}\right|^{2}}{P}\right) \text {. }
$$

For $\Omega$ to become imaginary, we require $K<Q\left|A_{0}\right|^{2}$ so that the nonlinear cutoff wave number is obtained to be

$$
k_{n}=k_{c}\left[1+0.860\left|A_{0}\right|^{2} \varepsilon^{2}\right]-\frac{\varepsilon^{2}}{x_{2}} \int^{x_{2}} R\left(x_{2}^{\prime}\right) d x_{2}^{\prime} .
$$

The nonlinearity changes slightly the range of unstable wave numbers within the fluid slab. The band width of spectrum is $O\left(\varepsilon^{2}\right)$ in the wave number space for standing waves. Moreover, the band width of spectrum in the frequency space is $O(\varepsilon)$. It may be noted that the amplitude of free surface elevation taken by Tassoul and Dedic [3] is $2|A|$ in our notation. On setting $R=0$, which corresponds to assuming their initial conditions, results similar to those they obtained can be recovered.

The term $P\left(\partial^{2} A / \partial t_{1}^{2}\right)$ in Eq. (39) is essential to describe the long-time asymptotic behavior of wave modulation. Physically this plays the role of checking the steepening of the wave form. Moreover, there is a competition between nonlinearity dispersion effects, giving rise to the solution of the form:

$$
\left.A\left(x_{2}, t_{1}\right)=a\left(t_{1}\right) \exp \left(i \mu x_{2}\right) \exp |-i|^{x_{2}} R\left(x_{2}^{\prime}\right) d x_{2}^{\prime}\right\},
$$

where $\mu$ is a real constant and $a\left(t_{1}\right)$ is a complex function of $t_{1}$. Setting $a\left(t_{1}\right)=$ $\rho\left(t_{1}\right) \exp \left(i \sigma\left(t_{1}\right)\right)$ where $\rho$ and $\sigma$ are real functions of $t_{1}$, Eq. (39) yields

$$
\frac{1}{2}\left(\frac{d \rho}{d t_{1}}\right)^{2}+U(\rho)=E
$$


with

$$
U(\rho)=\frac{M^{2}}{2 \rho^{2}}-\frac{\mu \rho^{2}}{2 P}-\frac{Q \rho^{4}}{4 P},
$$

and

$$
M=\rho^{2}\left(d \sigma / d t_{1}\right) .
$$

This is equivalent to the classical equation of motion for unit mass with total energy $E$ and potential energy $U(\rho)$. The solution of (48) can be expressed in terms of Jacobian elliptic functions. Following Hasimoto and Ono [5] and Kakutani and Sugimoto [6], one can derive both solitary wave (known as "envelope soliton") and phase jumps as special cases when $M=0$. For the case $M \neq 0$, Eq. (48) can be integrated to furnish the solution in terms of Jacobian elliptic functions. This includes a depressive solitary wave as a particular case. This type of envelope soliton is referred as "dark soliton" in nonlinear optics.

\section{REFERENCES}

[1] R. S. Oganesian, Gravitational instability of a layer relative to two-dimensional transverse disturhances. Astron. Zh. 37, 458 (1960)

[2] B. B. Chark raborty, Stability of gratitating fluid layer of uniform thickness in presence of Coriolis force and a magnetic field, Indian J. Phys. 38, 490 (1964)

[3] J. L. Tassoul and H. Dedic, Finite amplitude disturbances in self-gravitating media, Astron. Astrophys. 26, 79 (1973)

[4] G. B. Whitham, Linear and non-linear waves, Wiley, New York, 1973

[5] H. Hasimoto and H. Ono, Non-linear modulation of gratity waves, J. Phys. Soc. Japan 33, 805 (1972)

[6] T. Kakutani and N. Sugimoto, Krylor-Bogoliubot-Mitropolsky method for non-linear wates, Phys. Fluids 17, 1617 (1974) 